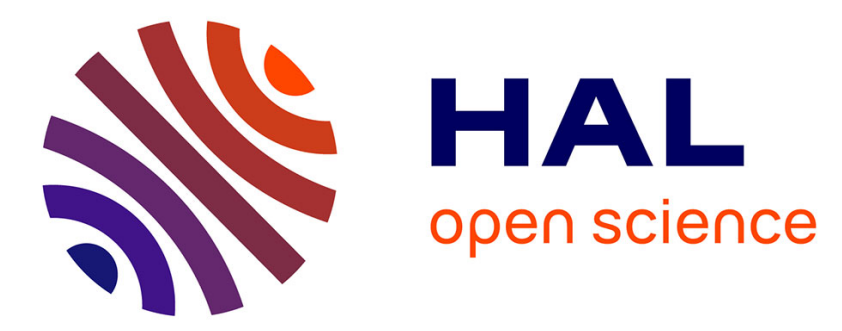

\title{
"If you've gone straight, now, you must turn left" - Exploring the use of a tangible interface in a collaborative treasure hunt for people with visual impairments
}

Quentin Chibaudel, Wafa Johal, Bernard Oriola, Marc J.-M. Macé, Pierre Dillenbourg, Christophe Jouffrais, Valérie Tartas

\section{To cite this version:}

Quentin Chibaudel, Wafa Johal, Bernard Oriola, Marc J.-M. Macé, Pierre Dillenbourg, et al.. "If you've gone straight, now, you must turn left" - Exploring the use of a tangible interface in a collaborative treasure hunt for people with visual impairments. ASSETS'20, 2020, Athens, Greece. 10.1145/3373625.3417020 . hal-02921663

\section{HAL Id: hal-02921663 https://hal.science/hal-02921663}

Submitted on 25 Aug 2020

HAL is a multi-disciplinary open access archive for the deposit and dissemination of scientific research documents, whether they are published or not. The documents may come from teaching and research institutions in France or abroad, or from public or private research centers.
L'archive ouverte pluridisciplinaire HAL, est destinée au dépôt et à la diffusion de documents scientifiques de niveau recherche, publiés ou non, émanant des établissements d'enseignement et de recherche français ou étrangers, des laboratoires publics ou privés. 


\section{"If you've gone straight, now, you must turn left" - Exploring the use of a tangible interface in a collaborative treasure hunt for people with visual impairments}

\author{
Quentin Chibaudel \\ IRIT, Université Paul Sabatier, \\ Toulouse, France, \\ quentin.chibaudel@irit.fr and CLLE, \\ Université Toulouse Jean Jaurès, \\ Toulouse, France \\ Marc J-M Macé \\ IRIT, CNRS, Toulouse, France \\ marc.mace@irit.fr
}

\author{
Wafa Johal \\ School of Computer Science and \\ Engineering, Faculty of Engineering, \\ University of New South Wales, \\ Sydney, Australia \\ wafa.johal@unsw.edu.au \\ Pierre Dillenbourg \\ CHILI, EPFL, Lausanne, Switzerland \\ pierre.dillenbourg@epfl.ch \\ Christophe Jouffrais \\ IPAL, CNRS, Singapore, \\ SingaporeIRIT, CNRS, Toulouse, \\ France \\ christophe.jouffrais@irit.fr
}

\author{
Bernard Oriola \\ IRIT, CNRS, Toulouse, France \\ bernard.oriola@irit.fr
}

\begin{abstract}
Tangible User Interfaces (TUI) have been found to be relevant tools for collaborative learning by providing a shared workspace and enhancing joint visual attention. Researchers have explored the use of TUIs in a variety of curricular activities and found them particularly interesting for spatial exploration. However, very few studies have explored how TUIs could be used as a collaborative medium for people with visual impairments (VIs). In this study, we investigated the effect of tangible interaction (a small tangible robot) in a spatial collaborative task (a treasure hunt) involving two people with VIs. The aim was to evaluate the impact of the design of the TUI on the collaboration and the strategies used to perform the task. The experiment involved six dyads of people with VIs. The results showed that the collaboration was impacted by the interaction design and open interesting perspectives on the design of collaborative games for people with VIs.
\end{abstract}

\section{CCS CONCEPTS}

- Human-centered computing: Interaction design: Empirical studies in interaction design; • Human-centered computing: Accessibility: Accessibility technologies; - Applied computing: Education: Collaborative learning; • Human-centered computing: Human computer interaction (HCI): Interaction devices: Haptic devices;

ACM acknowledges that this contribution was authored or co-authored by an employee, contractor or affiliate of a national government. As such, the Government retains a nonexclusive, royalty-free right to publish or reproduce this article, or to allow others to do so, for Government purposes only.

ASSETS '20, October 26-28, 2020, Virtual Event, Greece

(c) 2020 Association for Computing Machinery.

ACM ISBN 978-1-4503-7103-2/20/10 . \$ \$15.00

https://doi.org/10.1145/3373625.3417020

\section{KEYWORDS}

Spatial cognition, Robots, Non-visual interaction, Haptics, Maps, 3D model, Wayfinding, Learning, Game

ACM Reference Format:

Quentin Chibaudel, Wafa Johal, Bernard Oriola, Marc J-M Macé, Pierre Dillenbourg, Valérie Tartas, and Christophe Jouffrais. 2020. "If you've gone straight, now, you must turn left" - Exploring the use of a tangible interface in a collaborative treasure hunt for people with visual impairments. In The 22nd International ACM SIGACCESS Conference on Computers and Accessibility (ASSETS '20), October 26-28, 2020, Virtual Event, Greece. ACM, New York, NY, USA, 10 pages. https://doi.org/10.1145/3373625.3417020

\section{INTRODUCTION}

A Tangible User Interface (TUI) is defined as an interface that provides a "seamless coupling [between] everyday graspable objects [and] the digital information that pertains to them"[26]. Interaction based on TUIs is therefore not limited to the visual and auditory modalities, but also relies on touch [48] which is relevant for people with VIs [7]. Interestingly, TUIs are relevant tools to support collaborative activities and facilitate learning [13, 14], including applications in the domain of spatial cognition [27]. A large number of them can be classified as computer-supported learning environments [48]. They were found to be enjoyable and engaging for learners [9], which can have a positive impact on learning [49].

Collaborative activities are well adapted for teaching and learning in general $[14,17]$. Regarding spatial learning supported by collaborative activities, many studies focused on orientation and mobility for sighted people [e.g 44]. There are studies concerning the collaboration between VI and sighted people [55], but there are very few about the collaboration between people with VIs [4, 54]. 
In this paper, we explored a new collaborative setup involving people with VIs playing a spatial game. Collaborative problemsolving activities have shown to bring positive social and learning outcomes in education, and we wondered how to design such an activity for people with VIs. We specifically examined the potential of active tangibles as a medium of communication to share spatial information. Our contribution is not technically oriented but rather qualitative, about how graspable interactive robots (Active vs. Passive) may impact the collaboration between people with VIs.

\section{RELATED WORK}

\subsection{Tangible interaction for people with VIs}

Because touch and tactile (passive) interfaces are largely used by educators for VI students [28], research on TUIs for people with VIs has investigated different curricular fields. Tangible interfaces have been developed and evaluated to assist the exploration of diagrams [22], graphics [33], mathematics [2] and maps [6, 20]. For instance, Ducasse and colleagues $[17,18]$ developed a TUI to help people with VIs explore zoomable geographical maps. It has also been shown that $3 \mathrm{D}$ printed models can help people with VIs to learn about space [20,24]. Muhanad and Shakir (2011) developed a Multimodal Interactive Cubes for Object Orientation (MICOO) adapted for people with VIs [32]. It helped them to create and interact with diagrams and graphs. TUIs are of interest for people with VIs because they rely on manipulation and haptic feedback [18].

In the last decade, small graspable robots have been used to convey movement information in addition to their tangibility. For instance, Richter et al (2007) proposed RATI (Remote Active Tangible Interaction), a graspable robot used to improve distributive collaboration. It was used to visualize movement in a room [45] Another interesting example is the tangible mediated robot for teachers [19] that was used in educational activities with young children.

\subsection{Collaborative learning}

Collaborative learning can be defined as a learning situation during which participants actively contribute to a mutual learning goal and try to share the effort to reach this goal [46]. The "optimal collaborative effort" corresponds to an effort that helps participants to co-construct knowledge [15]. Currently, more and more collaborative learning activities are computer-supported [50]. TUIs are relevant tools to enhance collaborative learning activities [16].

The Cellulo is a graspable and tangible robot that can be held in someone's hand [41].It has been developed for education purposes of sighted pupils [42]. Several learning scenarios were developed, covering various areas of the curriculum and exploring its potential as a tool for collaborative learning. In meteorology [40], Cellulo robots were used on a map of Europe as haptic probes by students and used collaboratively to understand how wind is formed by high and low-pressure points. In another scenario targeting young children, two Cellulo robots were used in a team activity allowing them to explore the dynamic in writing cursive letters [23]. Recently, this activity was extended to collaborative exploration of simple shapes and for collaboration between sighted and children with visual impairments [38]. Other works have looked at systems that could enhance collaboration of people with VIs. Balata et al. [4] focused on navigation and showed that collaborative navigation and repeated exploration of an environment is useful to learn a pathway. Another study by Vallée and colleagues [54] indicated the interest of digital tools to improve collaboration between teenagers with VI. Their results showed that a tactile device used in collaboration by two people with VIs is relevant to share a common representation of the situation and solve a spatial task.

\subsection{Visual impairment and spatial learning}

Vision is important to construct cognitive maps [53] that can rely on two different reference frames: allocentric and egocentric mental representations [3, 25]. Even with few or no visual information, people with VIs can build cognitive maps, relying on other modalities such as movement, touch [21], audition [8] and verbal descriptions [37]. But people with VIs tend to use egocentric representations more than allocentric ones. For instance, it has been shown that they prefer using egocentric references to describe space [11]. One important question is: "will blind people still show a benefit in a condition that favors an egocentric strategy if they have the possibility of developing simultaneous perception based on (intrinsically superior) allocentric cues?" [11]. A possibility to address that question is to support different types of spatial learning in people with VIs, essentially in switching from maps to real settings and vice versa. TUIs can be used to make models of spatial settings and support the switch from egocentric to allocentric references, as well as sharing knowledge about certain places (e.g. landmarks and routes).

\section{STUDY}

\subsection{Brainstorming and design of the game}

Preliminary discussions with special education teachers raised that collaborative spatial games with peers may enhance motivation in learning spatial skills. Similar observations have been reported in different recent studies. For example, Balata et al. [4] focused on a navigation system based on collaboration between people with VI; Metatla et al. [39] worked on the co-design of a spatial game including sighted and VI students; Bonani et al. [5] and Correia et al. [12] both studied the interest of collaboration between robots and blind people; Pires et al [43] and Thieme et al. [52] both explored the interest of accessible computer programming with robot for children with VI. In line with our observations, these studies mention that robots, thanks to their capacity of autonomous movement and feedback (sound, haptic), are particularly adapted for VI people to work on spatial skills. They also show that they are versatile enough to be used in educational settings.

However, these papers were focused on the collaboration between one user and the robot. For instance, in the study proposed by Köhlmann [29], it is mentioned that "collaboration is mostly used in face-to-face situations and online-based computer supported collaboration with more than one blind participant is rare". In this study, based on these previous works and on this last observation, we aimed to develop a game that relies on a robot as a mediation tool for collaborative activity between two people with VI.

Different robots were relevant for this type of experiment [36, 38, 43]. Among these robots, the Cellulo was the lightest with 168 $\mathrm{g}$ [41] when the others were about 800 to $900 \mathrm{~g}$ [43]. In addition, 
the Cellulo is quite small: $7.5 \mathrm{~cm}$ from edge to edge and $3 \mathrm{~cm}$ high [41]. The other robots are much taller: between 15 and $30 \mathrm{~cm}$ high and 18 and $30 \mathrm{~cm}$ long [43]. We chose the Cellulo because it is easy to grasp and it has been shown that it can convey haptic feedback to both adults and children [40, 42].

Before starting the design sessions, we met two special education teachers to talk about the interest of multimodal interaction and graspable robots to design collaborative games. They quickly confirmed their interest, especially in the spatial domain. They acknowledged the interest of creating pairs of collaborators that can play independently (without the assistance of a sighted person). Following this first session, we did a simple demo session aiming at showing the Cellulo robots to one of the two teachers and her class of six pupils with mixed visual abilities. All of them were enthusiastic about the robots and serious games relying on it. Following these sessions, we designed a collaborative treasure hunt as a proof of concept, including the Cellulo as a medium to collaborate and share spatial information.

\subsection{General goal and hypothesis}

The study consisted in a treasure hunt involving two people with VIs. Each participant had a specific role: guide or explorer. The explorer moves in a large remote room where five clues and a treasure are hidden. The guide explores a map (2.5D model) representing the real settings and indicating the position of the clues and treasure. The explorer's location is indicated on the map by a Cellulo. The guide and explorer must collaborate by talking to each other in order to find the clues and, finally, the treasure (see Figure 1).

The task included two conditions depending on the functions attributed to the Cellulo. In the Active condition, the Cellulo reflected the explorer's location and orientation in real time. In the Passive condition, the Cellulo was not moving alone, but it could be used by the guide as a tool to represent the ongoing location and orientation of the explorer in the room.

The aim of the study was to evaluate the impact of the Active vs. Passive Cellulo on the game performance, as well as on the type of collaboration between them. We notably explored how these two modalities (Active vs. Passive) reshape the different aspects of collaboration (joint attention, communication, consensus reaching). As the Active Cellulo provides ongoing information about the explorer's location in the room, it should help the guide to give explicit instructions to the explorer. Hence, we hypothesized that: 1) the Active Cellulo should ease the task and decrease the time needed to find the treasure, and 2) it should help the collaborators to reach a collaborative optimal effort and hence better co-construct knowledge about the explored space.

\subsection{Participants}

Twelve people with visual impairments ( 8 males and 4 females, divided in 6 dyads) participated to the study. They did not show any other sensory or cognitive impairments. Their mean age was 36.5 years old (SD: 8.7 years). Among these people, 1 was early blind, 1 was late blind, 5 had low vision from birth, and 5 became visually impaired later (see Table 1). Participants scored their use of Information and Communication Technologies (computer, smartphone, tablet) and tactile maps on a 5-level Likert scale from 1 (almost never) to 5 (very often). The mean score for the use of ICT was 3.92 $(\mathrm{SD}=1.13)$ and the mean score for the use of tactile maps was 1.25 $(\mathrm{SD}=0.46)$.

\subsection{Experimental setting}

The room to be explored was a rectangular shape of $7.5 \times 15$ meters including 13 chairs. The positions of the 13 chairs were fixed in each condition. The guide was provided with a $2.5 \mathrm{D}$ map of the explored room made with a tactile line for the walls and foam objects representing the 13 chairs. The starting point and one clue at a time (little rubber ball stuck on one foam chair) were indicated on the map too (see Figure 1). To make sure that the configuration of the chairs did not bias the experiment, the layout for the second condition was a mirror image of the first one and hence with similar complexity (see Figure 2).

A "landmark chair" was located against the wall in the exploration room (red square in Figure 2). This chair was introduced during preliminary testing of the protocol. Indeed, in many cases, in the Passive condition, the explorer was lost in the room and could not provide the guide with any valid cues to get located. With the landmark chair, it was easy to solve that problem. When the explorer was lost, he could follow the wall until he reaches this unique chair. Participants were told about this landmark chair before starting the game.

The location and orientation of the explorer on the map was represented by a Cellulo (small graspable robot containing motors and three wheels). The Cellulo that we used in this study was augmented with a tactile cue on one side, which indicated the orientation of the explorer in the room (see Figure 1).

Guide and explorer both received a phone with a bone conduction headset, which allowed communicating without impeding audition and having the hands free to explore the environments.

\subsection{Task}

At the beginning of the session, the guide was in front of the $2.5 \mathrm{D}$ map, and the explorer was taken to the starting point in the room. The first clue was pinned on the map and an object was dropped off on the corresponding chair in the room. Then the guide had to lead the explorer from the starting position to the first clue. He could provide both egocentric (e.g. "do one step on the left") and allocentric (e.g. "there are two chairs side by side") instructions to accurately guide the explorer. In case of disorientation, they had to collectively find a solution to get reoriented. After finding the first clue, the second one was positioned, and they had to continue the game. In each condition, the clues order was the same (see numbers in Figure 2). Neither the guide nor the explorer knew the positions of the clues in advance. The game stopped after finding the fifth clue (i.e. treasure). They were told to achieve the treasure hunt as quickly as possible because time was recorded. If it took longer than five minutes to find a single clue, they received a penalty and the experimenter helped them to find the clue.

\subsection{Conditions}

In the Active condition, the Cellulo represented the location of the explorer in real-time. In the Active Cellulo condition, the guide always knows the location and orientation of the explorer in the 
Table 1: Participants.

\begin{tabular}{|c|c|c|c|c|c|c|}
\hline & Role & Gender & Age & Situation & Locomotion tool & During the experiment \\
\hline \multirow[t]{2}{*}{ Dyad 1} & Guide & Male & 46 & Low Vision late & Cane & No \\
\hline & Explorer & Male & 34 & Blind late & Dog or cane & Cane \\
\hline \multirow[t]{2}{*}{ Dyad 2} & Guide & Male & 42 & Blind late & Cane & No \\
\hline & Explorer & Male & 27 & Low vision early & Cane & Cane \\
\hline \multirow{2}{*}{ Dyad 3} & Guide & Male & 21 & Low Vision early & Cane & No \\
\hline & Explorer & Female & 25 & Low Vision early & Cane & No \\
\hline \multirow[t]{2}{*}{ Dyad 4} & Guide & Female & 35 & Low Vision late & No & No \\
\hline & Explorer & Male & 44 & Low Vision late & Cane & No \\
\hline \multirow[t]{2}{*}{ Dyad 5} & Guide & Female & 40 & Low Vision late & No & No \\
\hline & Explorer & Male & 44 & Low Vision early & Cane & No \\
\hline \multirow[t]{2}{*}{ Dyad 6} & Guide & Male & 55 & Low Vision early & Cane or dog & No \\
\hline & Explorer & Female & 25 & Low Vision late & No & No \\
\hline
\end{tabular}

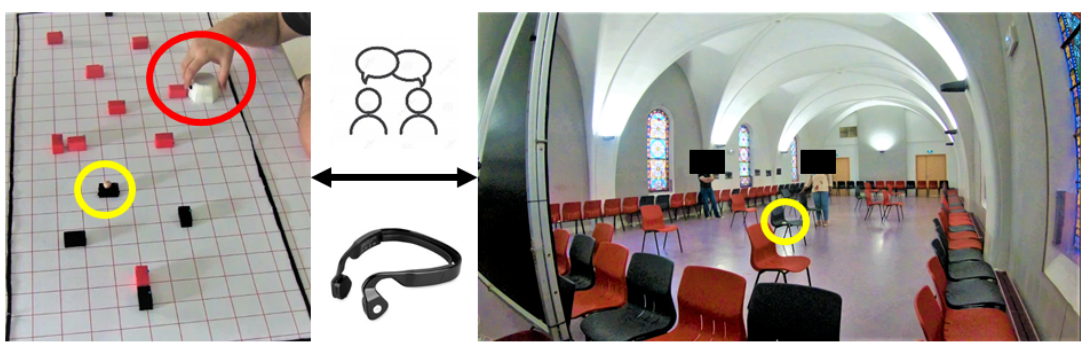

Figure 1: 2.5D map (left) and exploration room (right). Left: Guide exploring the 2.5D map with the chairs (foam cubes), clue (pin on a foam cube; yellow circle) and Cellulo (red circle). The Cellulo is a graspable robot with a form of hexagonal shape, measures $7.5 \mathrm{~cm}$ from edge to edge, $3 \mathrm{~cm}$ high and weights $167.8 \mathrm{~g}$. For more technical precision about it(see [41] for details). Right: the explorer (in the middle of the picture) is following the instructions provided by the guide to find the clue (object on a chair; yellow circle). The second person in the room is an experimenter in charge of safety and positioning the clues.

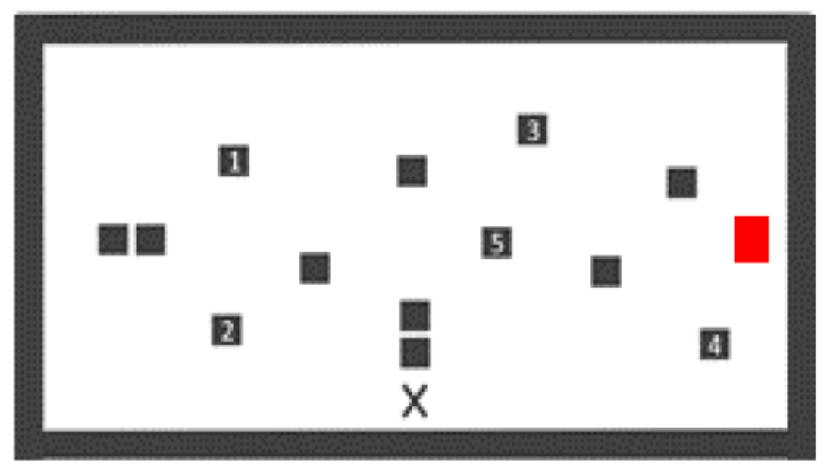

Figure 2: Map of the exploration room. Each square represents a chair. The positions of the five clues are indicated with numbers. The five clues are not set up at the beginning of the task. "Clue 2" is added when "clue 1 " has been found. The landmark chair is indicated with the red color. The cross shape represents the explorer's starting point.

room. In the Passive condition, the Cellulo was provided to the guide as a passive tool to represent the location and orientation of the explorer. The order of the Active and Passive conditions was counterbalanced across dyads.

In our experimental setup, tracking the explorer's location in real time was technically challenging (whole body tracking in the room and real-time communication of the coordinates to the Cellulo). Because we only targeted a proof of concept experiment, we used a "Wizard of Oz" method to move the robot in the Active condition. The wizard, an experimenter, was looking at the room video and moved the Cellulo according to the explorer's location, without interfering with the guide's hand movement. Participants did not mention any issues related to this method. They played the game and used the Cellulo as if it was moving by itself (see the results and limitations sections).

\subsection{Protocol}

The experiment was conducted as follows:

1. Presentation of the experiment (aim of the experiment and signature of the consent form),

2. Collection of personal details: gender, age, visual impairment, use of technologies and tactile maps,

3. First condition (e.g. Active): five clues must be found as quickly as possible in a specific order (see Figure 2), 
Table 2: Meier's grid

\begin{tabular}{ll}
\hline Dimension & Factor(s) to evaluate the dimension \\
\hline Communication & $\begin{array}{l}\text { Sustaining mutual comprehension: participants make their contribution understandable } \\
\text { Dialog management: there is an alternation of dialogs, with clear questions, etc. }\end{array}$ \\
Joint information & $\begin{array}{l}\text { Information pooling: process to elicit and externalize information } \\
\text { processing }\end{array}$ \\
Reaching consensus: participants treat information and make an evaluation to make a relevant decision \\
Task division: management of sub-tasks dependencies by participants \\
Time management: how participant cope with time dimension \\
Technical coordination: how participant cope with technical interdependencies \\
Reciprocal information: equality between participants in contributing to problem solving and decision \\
Interpersonal & making \\
relationship & Individual task orientation (participant 1): measurement of the first participant motivation \\
Motivation & Individual task orientation (participant 2): measurement of the second participant motivation \\
&
\end{tabular}

4. At the end of this first block, the guide had to answer a user experience questionnaire (MeCue [31]) and the explorer had to relocate the five clues on the $2.5 \mathrm{D}$ map,

5. Repetition of the steps 3 and 4 with the other condition (Passive in this example).

\subsection{Data and statistics}

3.8.1 Interaction with the robot. For each condition, based on video of the guide, interaction between the guide and the robot was evaluated according two criteria: the number of times he used the robot and the time spent using the robot.

3.8.2 Performance and localization of the clues on the 2.5 D map. For each condition, the performance was evaluated with two criteria: time (to find each clue and to achieve the whole treasure hunt) and total number of clues found. The accuracy of the localization by the explorer was assessed with the number of clues that were correctly located on the map (number and location).

3.8.3 User experience. In the MeCue [31], there are 30 items divided into four independent factors: product perception, emotions, consequences, and global evaluation. For the modules 1 to 3 , users must rate each item on a 7-level Likert scale going from «strongly disagree »to «strongly agree ». The fourth module is a global evaluation by the user from -5 to +5 .

3.8.4 Assessment of the collaboration. Dialogs between the two partners were recorded and transcribed. Three independent annotators evaluated the collaboration according to the Meier et al.'s grid [34] including five dimensions divided in different factors (see Table 2). Annotators were provided with the transcriptions and a "rating handbook" (i.e. a document that explains how to evaluate the collaboration, see [34]). For each condition and each factor, each judge gave a score going from "very bad" (score of -2) to "very good" $($ score +2$)$.

3.8.5 Statistics. Descriptive and inferential statistics were done with R studio (version 1.1.463 for Windows 10). A Shapiro test was used to check that the distributions were normal. According to normality, either a T-test or a Wilcoxon test was used to assess differences between the Active and Passive conditions.

\section{RESULTS}

\subsection{Interaction with the robot}

Depending on the condition (Active vs. Passive), the guide interacted differently with the Cellulo. Table 3 presents, for each dyad and according to each condition, the number of robot touches and the average handling time.

From these results, three types of interaction can be distinguished. The guide of the dyad \#3 never used the Cellulo whatever the condition. This guide had a low vision (see Table 1 for participant's characteristics) and he was able to follow the robot's location by sight in the Active condition. He adapted his instructions to the explorer accordingly. In the Passive condition, he did not used the robot, but instead used his own hand to represent the explorer's location.

The guide of the dyad \#6 has a low vision too. In the Active condition, as the guide \#3, he was able to follow the robot's location by sight and adapted the instructions accordingly. He only touched the robot a few times to determine the orientation of the explorer. He was more interested in using the Cellulo in the Passive condition because he wanted to try. He said that "it's funny to move it at the same time as the explorer's movement", which may explain the difference with the other dyads. However, the explorer got lost to find the last clue. At that time, the guide stopped using the robot and used his own hand to try to solve the problem. This behavior eventually shows that the Cellulo was "fun" to use, but when the situation is getting harder, it represents another element to deal with, which makes the task more difficult.

Finally, the guides \#1, 2, 4 and 5 present a similar behavior. In the Passive condition, they used the Cellulo mainly at the beginning of the session. But, similarly to the guide \#6, when the explorer got lost, they stopped using it. They used the robot more often and for a longer time in the Active condition. They kept their hand on the robot to be sure that the explorer executes the instructions correctly and goes to the right direction.

\subsection{Task performance and localization of the clues}

In the Active condition, all the clues were found by all the dyads. In the Passive condition, three dyads found all the clues, and two 
Table 3: Interaction between the guide and the Cellulo

\begin{tabular}{lllll}
\hline & \multicolumn{2}{l}{ Number of touches } & \multicolumn{2}{l}{ Average duration of each touch $(\mathrm{s})$} \\
\cline { 2 - 5 } & Passive & Active & Passive & Active \\
\hline Dyad 1 & 2 & 8 & 3.4 & 14 \\
Dyad 2 & 4 & 22 & 2.6 & 6.8 \\
Dyad 3 & 0 & 0 & 0 & 0 \\
Dyad 4 & 19 & 42 & 7.9 & 8 \\
Dyad 5 & 1 & 8 & 2.3 & 7.9 \\
Dyad 6 & 51 & 3 & 10.5 & 2 \\
\hline
\end{tabular}

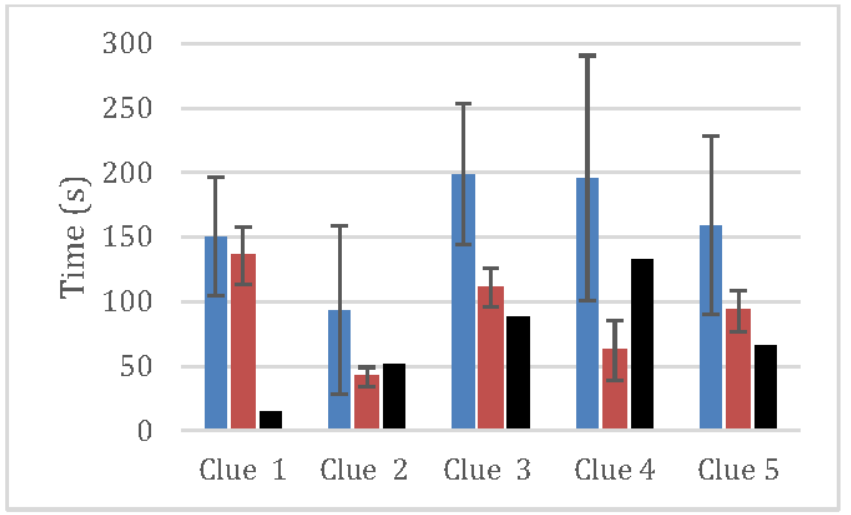

Figure 3: : average performance (in seconds) for each clue according to the condition. Blue: Passive condition; Red: Active condition; Black $=$ difference of time (Passive-Active)

dyads found 4 clues out of 5 . The post experiment test administered to the explorers showed that they could correctly localize on the map more clues in the Passive condition compared with the Active (respectively 1.67 vs 0.33 on average; Wilcoxon test, $\mathrm{p}=0.054$ ). In the Active condition, the task lasted $531 \pm 33$ seconds in average. In the Passive condition the task lasted $955 \pm 131$ seconds in average. Figure 3 presents the average times and standard deviations (in seconds) to find each clue according to the condition. On average, it takes longer to find a clue in the Passive condition than in the Active condition, which shows that it is more difficult to find a clue in the Passive condition. The Wilcoxon test showed that the difference between the two conditions is significant (Wilcoxon-test, $\mathrm{p}=0.045)$.

In addition, Figure 3 shows that the standard deviation in the Active condition is always small and homogeneous (less than 50 s). It does not depend on the difficulty to find each clue. On the contrary, it is larger and depends on the clue number in the Passive condition (between 92 and 190 seconds). The difference of standard deviation according to the condition is significant (Wilcoxon test; $\mathrm{p}=0.008$ ). Interestingly, the time difference between Active and Passive conditions increases from the first ( 15 seconds) to the fourth (133 seconds) clue. Altogether, these results show that the dyads spent less time and were more efficient in finding the clues in the Active condition.

\subsection{User experience}

All the participants reported that playing the game was enjoyable, independently of being the guide or the explorer. The results of the MeCue were good and similar for both conditions. For instance, the global evaluation (final question of the MeCue) was rated $4.6 \pm 0.4$ and $4.2 \pm 0.3$ (on a scale from -5 to +5 ) in the Active and Passive conditions respectively, which are not significantly different. Not any dimension of the MeCue was found to be significantly different between the two conditions.

\subsection{Assessment of the collaboration}

We performed both quantitative and qualitative assessments of the collaboration. Results observed for the different dimensions of the Meier's grid are presented in Figure 4 and show that the collaboration between the guide and explorer is different according to the condition. Some of the factors reported in the Meier's grid are not impacted by the condition (e.g. sustaining mutual comprehension, dialog management, etc.), which reflects that the Active Cellulo does not have much effect on the dimensions of Communication and Coordination. On the contrary, the five factors included in the three other dimensions were significantly different: Joint Information Processing dimension (including Information Pooling (T-test, $\mathrm{p}=3,8.10^{-5}$ ) and Reaching Consensus (T-test, $\left.\mathrm{p}=1,7.10^{-6}\right)$ ); Interpersonal Relationship dimension (including Reciprocal Information (T-test, $\left.\mathrm{p}=1.5 .10^{-4}\right)$ ), and Motivation dimension (including Individual Task Orientation for the guide (T-test, $\mathrm{p}=0.01$ ) and for the explorer (T-test, $\left.\mathrm{p}=3.8 \cdot 10^{-3}\right)$ ).

From a qualitative point of view, different excerpts of dialogs in the Active vs. Passive conditions (additional examples are provided in the supplementary material) are presented to illustrate different ways of collaborating. Here is an example of the dialog in the Active condition:

- Guide: "Perfect. Now, you go all the way"

- Explorer: "How many steps now?"

- Guide: "You do seven ... eight steps."

- Explorer: "Seven or eight?"

- Guide: "Go. But large steps. You can go safely, trust me"

- Explorer: "one, two, three, four, five, six, seven, eight"

- Guide: "Go one more"

- Explorer: "Okay, let's go. Nine"

In this example, there was no attempt for each participant to make an equal contribution to collaborating or taking decision 


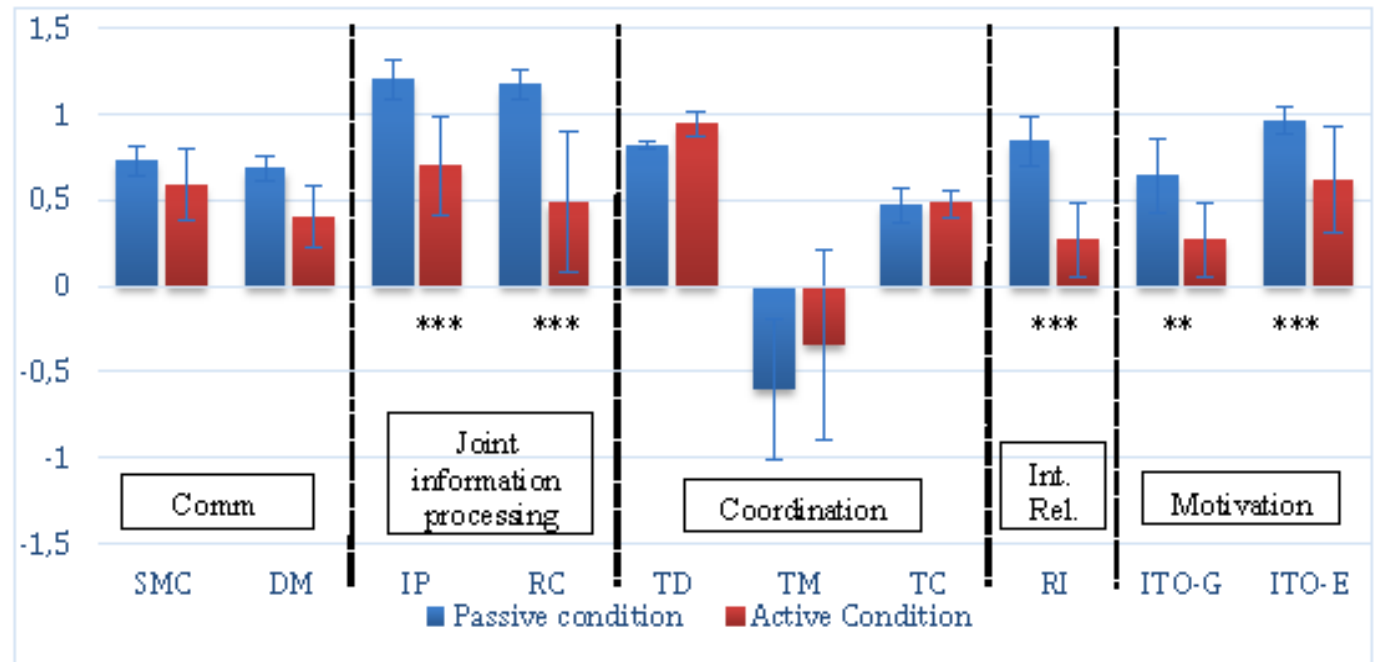

Figure 4: Scores for the different factors (and dimensions) of the collaboration $\left({ }^{*}<0.05,{ }^{* *}<0.01,{ }^{* * *}<0.001\right)$. SMC: Sustain Mutual Comprehension; DM: Dialog Management; IP: Information Pooling; RC: Reaching Consensus; TD: Task Division; TM: Time Management; TC: Technical Coordination; RI: Reciprocal Information; ITO-G: Individual Task Information - Guide; ITO-E: Individual Task Information - Explorer; Comm.: Communication; Int. Rel.: Interpersonal Relationship

together. The guide provides instructions without asking for additional information, and the explorer must follow. As indicated by the phrase "trust me"; the guide is leading the collaboration. In comparison, the following verbatim was extracted from the same dyad in the Passive condition:

- Guide: "If you have gone straight, now, you must turn left"

- Explorer: "But if I am going left, I am going into the wall ... On the left I cannot, there is the wall in front of me"

- Guide: "There is a wall in front of you?"

- Explorer: "In fact, to explain, I am in the left corner of the room. It means that on my left, there is a wall. And, in front of me, there is a wall too"

- Guide: "Are you in the left corner?"

- Explorer: "I cannot go straight, neither to the left. I can only go to the right"

- Guide: "You can only go to the right?"

- Explorer: "I am in the corner of the wall"

- Guide: "Okay. So, go to your right-hand side..."

In this example, there is a better repartition of responsibilities and a real need for the guide to understand where the explorer is and what he is doing. There is information pooling and the participants are trying to reach a common understanding of spatial information (e.g., the explorer's location in the room). This may explain why it is longer to solve the task in this condition.

The collaboration was also slightly impacted by the visual status of the participants. When VI people could detect visual features, they gave the information to their partner. For example, after an instruction, a VI explorer responded: "Yes I can, because I can see a little bit, but if you ask that to a blind person, he will be lost!". Blind explorers compensated using their long cane. A blind explorer was lost and said: "Wait, I am going to explore around the chair with my cane". The guide had doubts about the information provided by the blind explorer and answered: "Are you sure that you have well explored around you?". The notion of trust (included in the interpersonal relationship) was more important for blind people than for VI people.

Finally, strategies were influenced by the condition too. In the Passive condition, dyads developed a more global strategy. For instance, the guide first selected the easiest path for the explorer and then gave the instructions, which is illustrated in the following example: "Wait, I am thinking about the easiest path for you, the most appropriate, because it is a bit complicated". The explorer was understanding and responded "OK, I'm waiting". On the contrary, in the Active condition, it was a less global strategy with instructions adapted in real time. The following verbatim illustrates how the guide changed instructions while grasping the robot to assess the explorer's displacements: "Wait, there's a tactical error in my plan... Go to the second chair on your left".

\section{DISCUSSION}

\subsection{Impact of the design on the collaboration}

The study showed that the Active Cellulo helps the partners to achieve a better performance in the spatial game when compared to the Passive mode. 3D models are well adapted for VI people $[24,43]$ and interactivity provided by the Cellulo, similar to other technologies (see $[12,30,51]$ ), is relevant. Although the Cellulo could be of interest for representing the explorer location and orientation in the Passive condition, the guides experienced limitations in using it. As soon as a problem appears, they forget the Cellulo, and prefer using their own hand to represent the explorer's location. They also engaged in a more verbal collaboration. Only two dimensions of the collaboration, as defined by Meier et al. [34] were not impacted by the condition (Active vs. Passive): communication and coordination. In such a complex task for people with VI, both 
communication and coordination are crucial for the success of the collaboration [56]. Communication corresponds to trying to get a common and shared representation of the situation [10] and coordination is to make a plan on how to solve the case [34]. The collaborative game involved two persons who knew each other, and the roles were assigned at the beginning of the game.

The three other dimensions (joint information processing, interpersonal relationship and motivation) were impacted by the Active Cellulo. Joint information processing represents the importance for participants to process and share complementary knowledge about the spatial layout of the game. With the Active Cellulo, we observed series of instructions like: "Stop. Now, turn to your right. Now, you go straight", etc. On the contrary, when the Cellulo was Passive, they were many verbatims showing that the guide was missing information about the position of the explorer ("I cannot see you", "I cannot tell if you are good or not"). Interpersonal relationship evaluates the behavior of both partners during the game (respect for the other, tone of the speech, etc., see [34]). This dimension can depend on the (as)symmetry between the partners [14]. During the study, we did not observe any conflicts between the partners; probably because it was a game. But, the design of the interaction (Active vs. Passive Cellulo) influenced the relationship. In the Active condition, the robot cannot make any mistakes since it represents the localization and orientation of the explorer in real time. Then, both the guide and explorer can attribute mistakes to each other; which has an impact on the score of interpersonal relationship.

Finally, motivation illustrates the commitment of each person in solving the problem [34]. As previously mentioned, the guide always knows the explorer's position with the Active Cellulo and does not need additional feedback. This situation creates an asymmetry in the players' role. The guide is better informed and has a "superior" position. For example, a guide was saying "It will be very easy if you listen carefully to what I am saying". Then the explorer must follow the guidance instructions. This asymmetry has a negative impact on motivation, and probably more specifically on the explorer's motivation.

In conclusion, the results show that the Passive condition better supports an "optimal collaborative effort" with more attempts for each player to share his own representation of the situation and share relevant spatial information. On the contrary, the Active Cellulo turns the collaboration into a "guidance task" that improves the performance but decreases the collaborative process.

\subsection{Impact of the design on user experience}

Although the Active Cellulo decreases the challenge associated with the treasure hunt (the task is easier), there was no difference in the measured user experience according to the condition. This may be due to the design of the experiment: a treasure hunt is a pleasant game and people with VIs have very few opportunities to play such collaborative games. Hence, it appears that, whether Active or Passive, the type of Cellulo does not have much impact on the participants' overall experience.

\subsection{Importance of collaborative TUIs on learning and gaming}

The development of TUIs opens new opportunities and challenges in the field of collaboration $[1,44]$. In this work, we designed a collaborative game relying on tangible interaction and involving people with VIs. There are important lessons that we can learn from the study. First, the players with VI enjoyed collaborating in the aim of solving a complex spatial task. It is an important observation because spatial games that are accessible to people with VIs are rare. In addition, this game appeared as enjoyable and could be considered as a serious game aiming to improve spatial skills and to learn about a specific environment. Previous studies have shown that spatial games played in virtual environments can help to learn about space $[35,47]$. But these studies involved only one learner immersed in virtual reality, which may hinder inclusivity and social contacts. Our study shows that a collaborative game based on tangible interaction also fosters spatial skills and specific spatial knowledge (i.e. about a specific place). But interestingly, it can leverage on the participation of a tutor having visual impairments too, who better understand the problems and provides better instructions. It is in line with the observation that "there are many more visually impaired users with specific knowledge than there are instructors available" [4].

Another, interesting observation is that the design of the TUI can shape the type of collaboration. According to the need of the game designer (e.g. serious game if the designer is a special education teacher), the robot can provide different feedback and, hence, change the difficulty of the game, change the role assigned to each player, and change the type of skills and knowledge that is shared. The Active condition could for instance support the use of appropriate vocabulary (e.g. front, back, left, right) in children with VIs. The Passive condition may support the resolution of more complex spatial problems involving two teenagers with better verbal and spatial skills.

\subsection{Limitation of the study}

The Wizard of $\mathrm{Oz}$ method that we used may have slightly changed the results. Indeed, the behavior of the robots with this method was error-free, accurate and with very short delays. Hence, the trust accorded to the robot was greater, which may have an impact on joint information processing. However, according to our observations, if accuracy of the game is fair enough (e.g. less than $1 \mathrm{~m}$ ) and time lags short (e.g. less than $1 \mathrm{sec}$.), we do not envision any issues with real-time connected robots.

We observed a non-significant trend showing that the explorer achieves a better cognitive map of the room in the Passive condition. In that condition, each game is longer and problem solving depends on trial and errors, which may explain why the cognitive map is better. But another interpretation is that the dialog between players is richer in order to find a solution. However, we did not observe any evidence that the sharing of spatial information has significantly increased in the Passive condition. For instance, the number of allocentric and/or egocentric references observed in the verbatims (result not showed) was similar in both conditions. Then, the trend of a better cognitive map that we observed could be the result of an implicit learning related to longer exploration and trial and errors of 
each player individually. In addition, our sample of participants with VIs was limited (12 participants divided in 6 dyads) and included people with different visual abilities and different spatial skills. Thus, the results on spatial learning cannot be generalized and must be taken with care. These different hypotheses should be addressed in a specific study.

\subsection{Perspective and future work}

Currently, the Active Cellulo is working as a dynamic avatar providing the location and orientation of a remote collaborator. We can imagine more subtle interactions in order to design games that are getting gradually more and more challenging. For instance, we could imagine an intermediate mode to help the players to solve spatial problems. In this mode, the Cellulo would not move by itself but could be used as a probe to confirm guesses made by the guide. When the guide puts the Cellulo at the correct location on the map, there would be a signal (e.g. sound and/or haptic) confirming the spatial correspondence. Finally, we may also imagine an expert mode where the Active Cellulo provides false information about the location or orientation of the explorer, forcing the players to solve unexpected problems (of course, they should be aware of that possibility). Obviously, further work would be needed to check that increasing complexity has an impact on user experience and motivation, as well as spatial skills.

As we observed during the preliminary brainstorming sessions but also during follow-up discussions, special education teachers and orientation and mobility instructors were enthusiastic about tangible and multimodal interactive tools and collaborative games in their professional settings. They were convinced that such collaborative tangible games may fit their needs and improve spatial skills in people with VIs for four main reasons. Firstly, it increases motivation to learn about space, which can be crucial with early blind children or young adults. Secondly, players can switch their roles in the game and hence improve mental manipulation of egocentric and allocentric representations of space, which is an important spatial skill. Thirdly, it is based on the collaboration of two players with visual impairments who can fairly understand errors made by the other player and who can help with appropriate explanations and instructions. Finally, they can play independently, without the assistance of a sighted person, which relieves the fear of having the instructor repeatedly telling the same things.

In line with recent work [36, 38, 43], future work should address these assumptions and the development of a real-setting game.

\section{CONCLUSION}

The game that we designed in this study is a proof of concept resulting from brainstorming sessions organized with special education teachers. We showed that it allows to address specific knowledge (spatial skills in our case) but also transversal abilities such as collaborative problem solving (including perspective taking and argumentation). Depending on the interaction design, but also the age, the level of expertise of players and the educational aim, this kind of game can be adapted and address many use cases such as learning, resolution of problem or learn to collaborate with other peers. In accordance with [36, 43], we think that it is possible to generalize our results and to design educational and collaborative games with commercial robots for being used in real settings.

\section{REFERENCES}

[1] Luigi Atzori, Antonio Iera, and Giacomo Morabito. 2010. The Internet of Things: A survey. Computer Networks 54, 15: 2787-2805. https://doi.org/10.1016/j.comnet. 2010.05.010

[2] Mauro Ávila-Soto, Elba Valderrama-Bahamóndez, and Albrecht Schmidt. 2017. TanMath: A tangible math application to support children with visual impairment to learn basic arithmetic. ACM International Conference Proceeding Series Part F1285: 244-245. https://doi.org/10.1145/3056540.3064964

[3] Marios N. Avraamides, Jack M. Loomis, Roberta L. Klatzky, and Reginald G. Golledge. 2004. Functional equivalence of spatial representations derived from vision and language: Evidence from allocentric judgments. Journal of Experimental Psychology: Learning Memory and Cognition 30, 4: 801-814. https://doi.org/10. 1037/0278-7393.30.4.804

[4] Jan Balata, Jakub Franc, Zdenek Mikovec, and Pavel Slavik. 2014. Collaborative navigation of visually impaired. Journal on Multimodal User Interfaces 8, 2: 175185. https://doi.org/10.1007/s12193-013-0137-9

[5] Mayara Bonani, Raquel Oliveira, Filipa Correia, André Rodrigues, Tiago Guerreiro, and Ana Paiva. 2018. What My Eyes Can't See, A Robot Can Show Me: Exploring the Collaboration Between Blind People and Robots. In Proceedings of the 20th International ACM SIGACCESS Conference on Computers and Accessibility (ASSETS '18), 15-27. https://doi.org/10.1145/3234695.3239330

[6] Emeline Brulé, Gilles Bailly, Anke Brock, Annie Gentès, and Christophe Jouffrais. 2018. An Exploratory Study of the Uses of a Multisensory Map with Visually Impaired Children. Multimodal Technologies and Interaction 2, 3: 36. https://doi. org $/ 10.3390 / \mathrm{mti} 2030036$

[7] Emeline Brulé, Gilles Bailly, Anke Brock, Frédéric Valentin, Grégoire Denis, and Christophe Jouffrais. 2016. MapSense: Multi-Sensory Interactive Maps for Children Living with Visual Impairments. In International Conference for HumanComputer Interaction (CHI 2016), 445-457.

[8] Giulia Cappagli, Elena Cocchi, and Monica Gori. 2017. Auditory and proprioceptive spatial impairments in blind children and adults. Developmental Science 20, 3. https://doi.org/10.1111/desc.12374

[9] Carmen Chai, Bee Lau, and Zheng Pan. 2019. Hungry Cat-A Serious Game for Conveying Spatial Information to the Visually Impaired. Multimodal Technologies and Interaction 3, 1: 12. https://doi.org/10.3390/mti3010012

[10] Sheila Clark, Jennifer AS Chaffer, and Baldwin Van der Linden. 1996. Town and grown: a collaborative venture in grief education. Fournal of interprofessional care 10, 2: 133-146.

[11] Luca Latini Corazzini, Tinti Carla, Schmidt Susanna, Mirandola Chiara, and Cesare Cornoldi. 2010. Developing spatial knowledge in the absence of vision: allocentric and egocentric representation generated by blind people when supported by auditory cues. Psychologica Belgica 50, 3\&4: 327-334.

[12] Filipa Correia, Raquel Oliveira, Mayara Bonani, André Rodrigues, Tiago Guerreiro, and Ana Paiva. 2019. Exploring Collaborative Interactions Between Robots and Blind People. In 2019 14th ACM/IEEE International Conference on Human-Robot Interaction (HRI), 365.

[13] Sébastien Cuendet and Pierre Dillenbourg. 2013. The Benefits and Limitations of Distributing a Tangible Interface in a Classroom. 10th International Conference on Computer-Supported Collaborative Learning, CSCL, 1: 137-144.

[14] Pierre Dillenbourg. 1999. What do you mean by collaborative learning? Collaborative learning Cognitive and computational approaches 1, 6: 1-15. https: //doi.org/10.1.1.167.4896

[15] Pierre Dillenbourg, David Traum, and Daniel Schneider. 1996. Grounding in Multimodal Task-Oriented Collaboration. In Proceedings of the European Conference on Artificial Intelligence in Education, 401-407.

[16] Son Do-Lenh, Patrick Jermann, Sébastien Cuendet, Guillaume Zufferey, and Pierre Dillenbourg. 2010. Task Performance vs. Learning Outcomes: A Study of a Tangible User Interface in the Classroom. Sustaining TEL: From Innovation to Learning and Practice: 5th European Conference on Technology Enhanced Learning, EC-TEL 2010, Barcelona, Spain, September 28 - October 1, 2010. Proceedings: 78-92. https://doi.org/10.1007/978-3-642-16020-2_6

[17] Julie Ducasse. 2016. Construction and exploration of an interactive and collaborative map for the visually impaired. ACM SIGACCESS Accessibility and Computing, 114: 29-35. https://doi.org/10.1145/2904092.2904099

[18] Julie Ducasse, Marc J-M Macé, Marcos Serrano, and Christophe Jouffrais. 2016. Tangible Reels. In Proceedings of the 2016 CHI Conference on Human Factors in Computing Systems, 2186-2197. https://doi.org/10.1145/2858036.2858058

[19] Fernando Garcia-Sanjuan, Javier Jaen, Vicente Nacher, and Alejandro Catala. 2015. Design and evaluation of a tangible-mediated robot for kindergarten instruction. In Proceedings of the 12th International Conference on Advances in Computer Entertainment Technology - ACE '15, 1-11. https://doi.org/10.1145/2832932.2832952

[20] Stéphanie Giraud, Anke M. Brock, Marc J.M. Macé, and Christophe Jouffrais. 2017. Map learning with a 3D printed interactive small-scale model: Improvement of space and text memorization in visually impaired students. Frontiers in Psychology 8: 10. https://doi.org/10.3389/fpsyg.2017.00930 
[21] Monica Gori, Alessandra Sciutti, David Burr, and Giulio Sandini. 2011. Direct and indirect haptic calibration of visual size judgments. PLOS ONE 6, 10: 1-5. https://doi.org/10.1371/journal.pone.0025599

[22] Timo Götzelmann. 2018. Visually Augmented Audio-Tactile Graphics for Visually Impaired People. ACM Transactions on Accessible Computing 11, 2: 1-31. https: //doi.org/10.1145/3186894

[23] Arzu Guneysu Ozgur, Ayberk Özgür, Thibault Asselborn, Wafa Johal, Elmira Yadollahi, Barbara Bruno, Melissa Skweres, and Pierre Dillenbourg. 2020. Iterative Design and Evaluation of a Tangible Robot-Assisted Handwriting Activity for Special Education. Frontiers in Robotics and AI 7: 29.

[24] Leona Holloway, Kim Marriott, Matthew Butler, and Samuel Reinders. 2019. 3D Printed Maps and Icons for Inclusion. In The 21st International ACM SIGACCESS Conference on Computers and Accessibility - ASSETS '19, 183-195. https://doi.org/ $10.1145 / 3308561.3353790$

[25] Lobectomy Humans, Janet D Feigenbaum, and Robin G Morris. 2004. Allocentric Versus Egocentric Spatial Memory After Unilateral Temporal Lobectomy in Humans. Neuropsychology 18, 3: 462-472. https://doi.org/10.1037/0894-4105.18.3. 462

[26] Hiroshi Ishii and Brygg Ullmer. 1997. Tangible bits: towards seamless interfaces between people, bits and atoms. Proceedings of the ACM SIGCHI Conference on Human factors in computing systems: 234-241. https://doi.org/10.1145/258549. 258715

[27] Mi Jeong Kim and Mary Lou Maher. 2008. The impact of tangible user interfaces on spatial cognition during collaborative design. Design Studies 29, 3: 222-253. https://doi.org/10.1016/j.destud.2007.12.006

[28] Alan J Koenig and M Cay Holbrook. 2000. Foundations of education, Volume II Instructional strategies for teaching children and youths with visual impairments. ERIC.

[29] Wiebke Köhlmann. 2012. Identifying Barriers to Collaborative Learning for the Blind. In Computers Helping People with Special Needs. 84-91. https://doi.org/10. 1007/978-3-642-31522-0_13

[30] Varsha Koushik and Shaun K Kane. 2019. "It Broadens My Mind." In Proceedings of the 2019 CHI Conference on Human Factors in Computing Systems - CHI '19, 1-12. https://doi.org/10.1145/3290605.3300744

[31] Carine Lallemand and Vincent Koenig. 2017. "How could an intranet be like a friend to me?” - Why Standardized UX Scales Don't Always Fit. In Proceedings of the European Conference on Cognitive Ergonomics 2017, 9-16.

[32] Muhanad S Manshad, Enrico Pontelli, and Shakir J. Manshad. 2011. MICOO (multimodal interactive cubes for object orientation). In The proceedings of the 13th international ACM SIGACCESS conference on Computers and accessibilityASSETS '11, 261. https://doi.org/10.1145/2049536.2049597

[33] David McGookin, Euan Robertson, and Stephen S.a. Brewster. 2010. Clutching at Straws: Using Tangible Interaction to Provide Non-Visual Access to Graphs. In Proceedings of the 28th international conference on Human factors in computing systems - CHI '10, 1715-1724. https://doi.org/10.1145/1753326.1753583

[34] Anne Meier, Hans Spada, and Nikol Rummel. 2007. A rating scheme for assessing the quality of computer-supported collaboration processes. International fournal of Computer-Supported Collaborative Learning 2, 1: 63-86. https://doi.org/10.1007/ s11412-006-9005-x

[35] Lotfi B Merabet, Erin C Connors, Mark A Halko, and Jaime Sánchez. 2012. Teaching the blind to find their way by playing video games. PloS one 7, 9: e44958. https://doi.org/10.1371/journal.pone.0044958

[36] Oussama Metatla, Sandra Bardot, Clare Cullen, Marcos Serrano, and Christophe Jouffrais. 2020. Robots for Inclusive Play: Co-designing an Educational Game With Visually Impaired and sighted Children. In Proceedings of the $2020 \mathrm{CHI}$ Conference on Human Factors in Computing Systems, 1-13. https://doi.org/10. $1145 / 3313831.3376270$

[37] Takahiro Miura, Yuka Ebihara, Masatsugu Sakajiri, and Tohru Ifukube. 2011. Utilization of auditory perceptions of sounds and silent objects for orientation and mobility by visually-impaired people. In Conference Proceedings IEEE International Conference on Systems, Man and Cybernetics, 1080-1087. https://doi.org/10.1109/ICSMC.2011.6083818

[38] Isabel Neto, Wafa Johal, Marta Couto, Hugo Nicolau, Ana Paiva, and Arzu Guneysu. 2020. Using Tabletop Robots to promote Inclusive Classroom Experiences. In Proceedings of the 19th ACM Conference on Interaction Design and Children (\{IDC\} '20). https://doi.org/10.1145/3392063.3394439

[39] Metatla Oussama, Bardot Sandra, Cullen Clare, Serrano Marcos, and Jouffrais Christophe. 2020. Robots for Inclusive Play: Co-designing an Educational Game
With Visually Impaired and sighted Children. In Proceedings of the $2020 \mathrm{CHI}$ Conference on Human Factors in Computing Systems - to appear.

[40] Ayberk Özgür, Wafa Johal, Francesco Mondada, and Pierre Dillenbourg. 2017. Windfield: Learning Wind Meteorology with Handheld Haptic Robots. In Proceedings of the 2017 ACM/IEEE International Conference on Human-Robot Interaction, 156-165. https://doi.org/10.1145/2909824.3020231

[41] Ayberk Özgür, Wafa Johal, Francesco Mondada, and Pierre Dillenbourg. 2017. Haptic-Enabled Handheld Mobile Robots. In Proceedings of the 2017 CHI Conference on Human Factors in Computing Systems, 2449-2461. https://doi.org/10.1145/ 3025453.3025994

[42] Ayberk Òzgür, Séverin Lemaignan, Wafa Johal, Maria Beltran, Manon Briod, Léa Pereyre, Francesco Mondada, and Pierre Dillenbourg. 2017. Cellulo: Versatile Handheld Robots for Education. In Proceedings of the 2017 ACM/IEEE International Conference on Human-Robot Interaction - HRI '17, 119-127. https://doi.org/10.1145/ 2909824.3020247

[43] Ana Cristina Pires, Filipa Rocha, Antonio José De Barros Neto, Hugo Simão, Hugo Nicolau, and Tiago Guerreiro. 2020. Exploring Accessible Programming with Educators and Visually Impaired Children. In IDC'20. https://doi.org/10. 1145/3392063.3394437

[44] Samira Pouyanfar, Yimin Yang, Shu-ching Chen, Mei-Ling Shyu, and S. S. Iyengar. 2018. Multimedia Big Data Analytics. ACM Computing Surveys 51, 1: 1-34. https: //doi.org/10.1145/3150226

[45] Jan Richter, Bruce H. Thomas, Maki Sugimoto, and Masahiko Inami. 2007. Remote active tangible interactions. Proceedings of the 1st international conference on Tangible and embedded interaction - TEI '07: 39. https://doi.org/10.1145/1226969. 1226977

[46] Jeremy Roschelle and Stephanie D. Teasley. 1995. The Construction of Shared Knowledge in Collaborative Problem Solving. Computer Supported Collaborative Learning 128: 69-97. https://doi.org/10.1007/978-3-642-85098-1_5

[47] Jaime Sánchez, Mauricio Saenz, and Jose Miguel Garrido. 2010. Usability of a Multimodal Video Game to Improve Navigation Skills for Blind Children. ACM Transactions on Accessible Computing 3, 2: 1-29. https://doi.org/10.1145/1857920. 1857924

[48] Orit Shaer. 2009. Tangible User Interfaces: Past, Present, and Future Directions. Foundations and Trends ${ }^{\circledR}$ in Human-Computer Interaction 3, 1-2: 1-137. https: //doi.org/10.1561/1100000026

[49] David J. Shernoff, Mihaly Csikszentmihalyi, Barbara Schneider, and Elisa Steele Shernoff. 2014. Student Engagement in High School Classrooms from the Perspective of Flow Theory. In Applications of Flow in Human Development and Education. Springer Netherlands, Dordrecht, 475-494. https://doi.org/10.1007/978-94-0179094-9_24

[50] Gerry Stahl. 2005. Group cognition in computer-assisted collaborative learning. Journal of Computer Assisted Learning 21, 2: 79-90. https://doi.org/10.1111/j.13652729.2005.00115.x

[51] Xiang Zhi Tan, Elizabeth J. Carter, Samantha Reig, and Aaron Steinfeld. 2019. Go that way: Exploring supplementary physical movements by a stationary robot when providing navigation instructions. ASSETS 2019 - 21st International ACM SIGACCESS Conference on Computers and Accessibility: 299-311. https: //doi.org/10.1145/3308561.3353805

[52] Anja Thieme, Cecily Morrison, Nicolas Villar, Martin Grayson, and Siân Lindley. 2017. Enabling Collaboration in Learning Computer Programing Inclusive of Children with Vision Impairments. In Proceedings of the 2017 Conference on Designing Interactive Systems, 739-752. https://doi.org/10.1145/3064663.3064689

[53] Catherine Thinus-Blanc and Florence Gaunet. 1997. Representation of space in blind persons: vision as a spatial sense? Psychological bulletin 121, 1: 20-42. https://doi.org/10.1037/0033-2909.121.1.20

[54] Aurélie Vallée, Katia Rovira, and Loïc Deschamps. 2016. A study of collaboration via a digital space accessible to adolescents with visual impairment. British fournal of Visual Impairment 34, 1: 83-90. https://doi.org/10.1177/0264619615612525

[55] Fredrik Winberg and John Bowers. 2004. Assembling the senses: towards the design of cooperative interfaces for visually impaired users. In Proceedings of the 2004 ACM conference on Computer supported cooperative work-CSCW'04, 332. https://doi.org/10.1145/1031607.1031662

[56] Gwen M Wittenbaum, Sandra I Vaughan, and Garold Strasser. 2002. Coordination in Task-Performing Groups. In Theory and Research on Small Groups. Kluwer Academic Publishers, Boston, 177-204. https://doi.org/10.1007/0-306-47144-2 9 